\title{
Triple Parton Scatterings in High-Energy Proton-Proton Collisions
}

\author{
David d'Enterria $^{1}$ and Alexander M. Snigirev ${ }^{2}$ \\ ${ }^{1}$ CERN, EP Department, 1211 Geneva, Switzerland \\ ${ }^{2}$ Skobeltsyn Institute of Nuclear Physics, Lomonosov Moscow State University, 119991 Moscow, Russia
}

(Received 23 December 2016; published 23 March 2017)

\begin{abstract}
A generic expression to compute triple parton scattering cross sections in high-energy proton-proton $(p p)$ collisions is presented as a function of the corresponding single parton cross sections and the

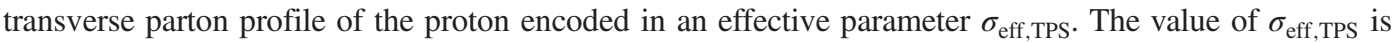
closely related to the similar effective cross section that characterizes double parton scatterings, and amounts to $\sigma_{\text {eff,TPS }}=12.5 \pm 4.5 \mathrm{mb}$. Estimates for triple charm $(c \bar{c})$ and bottom $(b \bar{b})$ production in $p p$ collisions at LHC and FCC energies are presented based on next-to-next-to-leading-order perturbative calculations for single $c \bar{c}, b \bar{b}$ cross sections. At $\sqrt{s} \approx 100 \mathrm{TeV}$, about $15 \%$ of the $p p$ collisions produce three $c \bar{c}$ pairs from three different parton-parton scatterings.
\end{abstract}

DOI: 10.1103/PhysRevLett.118.122001

The existence of multiparton interactions in high-energy hadronic collisions is a natural consequence of the finite size of hadrons, the fast increase of the parton flux at small parton longitudinal momentum fractions (Bjorken $x$ ), and the requirement of unitarization of the cross sections in perturbative quantum chromodynamics (pQCD) [1]. The field of multiparton interactions has attracted an increasing interest in the past years motivated, among other reasons, by the accumulated experimental evidence of double parton scattering (DPS) processes concurrently producing two independently identified hard particles in the same proton-(anti)proton $(p p, p \bar{p})$ collision, at Tevatron and LHC energies [2-6]. Multiple hard parton interaction rates depend chiefly on the degree of overlap between the matter distributions of the colliding hadrons [7], and provide valuable information on the poorly known transverse parton profile of the proton, the unknown energy evolution of the parton density as a function of impact parameter $(b)$, as well as on the role of many-parton correlations in the hadronic wave functions [8].

In this Letter, we present for the first time a quantitative estimate of the cross section for observing three separate hard interactions in a $p p$ collision [triple parton scattering (TPS)], a possibility considered earlier [9-11], but for which no simple formula of the expected rates based on the underlying single parton scattering (SPS) cross sections existed so far. A good understanding of TPS is not only useful to improve our knowledge of the $3 \mathrm{D}$ parton structure of the proton, but is also of relevance for a realistic characterization of backgrounds for rare final states with multiple heavy particles in

Published by the American Physical Society under the terms of the Creative Commons Attribution 4.0 International license. Further distribution of this work must maintain attribution to the author(s) and the published article's title, journal citation, and DOI. searches of new physics. As we show below, TPS can represent a non-negligible fraction of some particular final states at future hadron colliders such as FCC, projected to deliver several $100 \mathrm{fb}^{-1} / \mathrm{yr}$ integrated luminosities in $p p$ collisions at a c.m. energy of $\sqrt{s}=100 \mathrm{TeV}$ [12].

We review first the theoretical expression for TPS cross sections in a generic hadron-hadron collision, expressed as a convolution of SPS cross sections and generalized parton densities (dependent on $x, Q^{2}$, and $b$ ), and derive a simple factorized expression for the TPS cross sections as a triple product of SPS cross sections normalized by an effective cross section $\sigma_{\text {eff,TPS }}$ characterizing the transverse area of triple partonic interactions. From simple geometric considerations, we show how $\sigma_{\text {eff,TPS }}$ is closely connected to the effective cross section $\sigma_{\text {eff,DPS }}$ already measured in DPS. From the existing measurements of $\sigma_{\text {eff,DPS }}$ and realistic proton profiles, we derive the numerical value of $\sigma_{\text {eff,TPS, }}$, which proves very robust with respect to any particular choice of the underlying transverse parton density. As a concrete numerical example, we provide estimates for triple charm $(c \bar{c})$ and bottom $(b \bar{b})$ cross sections in $p p$ collisions at the LHC and FCC, based on next-to-next-to-leading-order (NNLO) calculations of the corresponding SPS cross sections.

In a generic hadron-hadron collision, the inclusive TPS cross section from three independent parton subprocesses $\left(h h^{\prime} \rightarrow a b c\right)$ can be written [9-11] as a convolution of generalized parton distribution functions (PDFs) and elementary cross sections summed over all involved partons:

$$
\begin{aligned}
\sigma_{h h^{\prime} \rightarrow a b c}^{\mathrm{TPS}}= & \frac{m}{3 !} \sum_{i, j, k, l, m, n} \int \Gamma_{h}^{i j k}\left(x_{1}, x_{2}, x_{3} ; \mathbf{b}_{\mathbf{1}}, \mathbf{b}_{2}, \mathbf{b}_{\mathbf{3}} ; Q_{1}^{2}, Q_{2}^{2}, Q_{3}^{2}\right) \\
& \times \hat{\sigma}_{a}^{i l}\left(x_{1}, x_{1}^{\prime}, Q_{1}^{2}\right) \hat{\sigma}_{b}^{j m}\left(x_{2}, x_{2}^{\prime}, Q_{2}^{2}\right) \hat{\sigma}_{c}^{k n}\left(x_{3}, x_{3}^{\prime}, Q_{3}^{2}\right) \\
& \times \Gamma_{h^{\prime}}^{l m n}\left(x_{1}^{\prime}, x_{2}^{\prime}, x_{3}^{\prime} ; \mathbf{b}_{1}-\mathbf{b}, \mathbf{b}_{2}-\mathbf{b}, \mathbf{b}_{\mathbf{3}}-\mathbf{b} ; Q_{1}^{2}, Q_{2}^{2}, Q_{3}^{2}\right) \\
& \times d x_{1} d x_{2} d x_{3} d x_{1}^{\prime} d x_{2}^{\prime} d x_{3}^{\prime} d^{2} b_{1} d^{2} b_{2} d^{2} b_{3} d^{2} b .
\end{aligned}
$$


In this expression, $\Gamma_{h}^{i j k}\left(x_{1}, x_{2}, x_{3} ; \mathbf{b}_{\mathbf{1}}, \mathbf{b}_{\mathbf{2}}, \mathbf{b}_{\mathbf{3}} ; Q_{1}^{2}, Q_{2}^{2}, Q_{3}^{2}\right)$ are the triple parton distribution functions, depending on the momentum fractions $x_{1}, x_{2}, x_{3}$ at transverse positions $\mathbf{b}_{\mathbf{1}}, \mathbf{b}_{\mathbf{2}}$, $\mathbf{b}_{\mathbf{3}}$ of the three partons $i, j, k$, producing final states $a, b, c$ at energy scales $Q_{1}, Q_{2}, Q_{3}$, with subprocess cross sections $\hat{\sigma}_{a}^{i l}$, $\hat{\sigma}_{b}^{j m}, \hat{\sigma}_{c}^{k n}$. The combinatorial prefactor $m / 3$ ! takes into account the different cases of (indistinguishable or not) final states: $m=1$ if $a=b=c ; m=3$ if $a=b$, or $a=c$, or $b=c$; and $m=6$ if $a, b, c$ are different. The triple parton distribution functions $\Gamma_{h}^{i j k}\left(x_{1}, x_{2}, x_{3} ; \mathbf{b}_{1}, \mathbf{b}_{2}, \mathbf{b}_{3} ; Q_{1}^{2}, Q_{2}^{2}, Q_{3}^{2}\right)$ encode all the parton structure information of relevance for TPS, and are typically assumed to be decomposable in terms of longitudinal and transverse components,

$$
\begin{aligned}
& \Gamma_{h}^{i j k}\left(x_{1}, x_{2}, x_{3} ; \mathbf{b}_{\mathbf{1}}, \mathbf{b}_{\mathbf{2}}, \mathbf{b}_{\mathbf{3}} ; Q_{1}^{2}, Q_{2}^{2}, Q_{3}^{2}\right) \\
& \quad=D_{h}^{i j k}\left(x_{1}, x_{2}, x_{3} ; Q_{1}^{2}, Q_{2}^{2}, Q_{3}^{2}\right) f\left(\mathbf{b}_{\mathbf{1}}\right) f\left(\mathbf{b}_{\mathbf{2}}\right) f\left(\mathbf{b}_{\mathbf{3}}\right),
\end{aligned}
$$

where $f\left(\mathbf{b}_{1}\right)$ describes the transverse parton density, often assumed to be a universal function for all types of partons, from which the corresponding hadron-hadron overlap function is derived:

$$
T(\mathbf{b})=\int f\left(\mathbf{b}_{\mathbf{1}}\right) f\left(\mathbf{b}_{\mathbf{1}}-\mathbf{b}\right) d^{2} b_{1} \text {, with } \int d^{2} b T(\mathbf{b})=1 .
$$

Making the further assumption that the longitudinal components reduce to the product of independent single PDFs, $D_{h}^{i j k}\left(x_{1}, x_{2}, x_{3} ; Q_{1}^{2}, Q_{2}^{2}, Q_{3}^{2}\right)=D_{h}^{i}\left(x_{1} ; Q_{1}^{2}\right) D_{h}^{j}\left(x_{2} ; Q_{2}^{2}\right)$ $D_{h}^{k}\left(x_{3} ; Q_{3}^{2}\right)$, the cross section of TPS can be expressed in the simple generic form

$$
\sigma_{h h^{\prime} \rightarrow a b c}^{\mathrm{TPS}}=\left(\frac{m}{3 !}\right) \frac{\sigma_{h h^{\prime} \rightarrow a}^{\mathrm{SPS}} \sigma_{h h^{\prime} \rightarrow b}^{\mathrm{SPS}} \sigma_{h h^{\prime} \rightarrow c}^{\mathrm{SPS}}}{\sigma_{\mathrm{eff}, \mathrm{TPS}}^{2}},
$$

i.e., as a triple product of independent single inclusive cross sections.

$$
\begin{aligned}
\sigma_{h h^{\prime} \rightarrow a}^{\mathrm{SPS}}= & \sum_{i, k} \int D_{h}^{i}\left(x_{1} ; Q_{1}^{2}\right) f\left(\mathbf{b}_{\mathbf{1}}\right) \hat{\sigma}_{a}^{i k}\left(x_{1}, x_{1}^{\prime}\right) \\
& \times D_{h^{\prime}}^{k}\left(x_{1}^{\prime} ; Q_{1}^{2}\right) f\left(\mathbf{b}_{\mathbf{1}}-\mathbf{b}\right) d x_{1} d x_{1}^{\prime} d^{2} b_{1} d^{2} b \\
= & \sum_{i, k} \int D_{h}^{i}\left(x_{1} ; Q_{1}^{2}\right) \hat{\sigma}_{a}^{i k}\left(x_{1}, x_{1}^{\prime}\right) D_{h^{\prime}}^{k}\left(x_{1}^{\prime} ; Q_{1}^{2}\right) d x_{1} d x_{1}^{\prime},
\end{aligned}
$$

normalized by the square of an effective TPS cross section,

$$
\sigma_{\mathrm{eff}, \mathrm{TPS}}^{2}=\left(\int d^{2} b T^{3}(\mathbf{b})\right)^{-1},
$$

which is closely related to the similar quantity

$$
\sigma_{\mathrm{eff}, \mathrm{DPS}}=\left(\int d^{2} b T^{2}(\mathbf{b})\right)^{-1},
$$

determined to be $\sigma_{\text {eff,DPS }} \simeq 15 \pm 5 \mathrm{mb}$ in DPS measurements at Tevatron and the LHC [5,13-16].

Whereas any TPS cross section can always be expressed as the triple product Eq. (1), with $\sigma_{\text {eff.TPS }}$ encoding all unknowns about the TPS dynamics, the geometrical interpretation of this latter quantity given by Eq. (3) relies on the assumption of the aforementioned simplifying yet economical assumptions on the factorization of longitudinal and transverse degrees of freedom, the absence of multiparton correlations, and flavor (gluon, quark)independent transverse parton profiles. In addition, there is no a priori reason to take the transverse hadron profile $f(\mathbf{b})$, and therefore also $\sigma_{\text {eff,TPS }}$ and $\sigma_{\text {eff,DPS }}$, as a constant with collision energy. As a matter of fact, DPS studies $[13,14,17]$ indicate that the experimentally extracted $\sigma_{\text {eff,DPS }} \simeq 15 \mathrm{mb}$ values are about a factor of 2 smaller (i.e., the DPS cross sections are about twice larger) than expected from Eq. (4) for a "standard" proton geometric profile. The concurrent measurement of $\sigma_{\text {eff,TPS }}$ and $\sigma_{\text {eff,DPS }}$ in different colliding systems and for different final states can help clarify all these open issues, shedding light on the 3D partonic structure of the proton and its evolution as a function of energy. Of course, achieving those goals will also require parallel theoretical developments in order to appropriately control the SPS contributions to final states with triple-hard (as well as DPS plus single-hard) particle production. In the case of double-SPS scatterings with charm states (plus electroweak bosons), calculation tools exist already today that show that carefully chosen kinematical cuts can reduce such SPS processes $[18,19]$.

To estimate the value of $\sigma_{\text {eff,TPS }}$, let us consider first a simplistic class of analytical models for the overlap function of a given hadronic $A A$ collision,

$T_{A A}(\mathbf{b})= \begin{cases}\frac{A^{2}(n+1)}{4 \pi R_{A}^{2}}\left\{1-\left[b /\left(2 R_{A}\right)\right]^{2}\right\}^{n} & b<2 R_{A} \\ 0 & b>2 R_{A},\end{cases}$

normalized to $\int d^{2} b T_{A A}(\mathbf{b})=A^{2}$, where exponents $n=$ $0,1, \infty$ give, respectively, a flat, hard-sphere-like, and Gaussian-like distribution in the interval $\left[0, R_{A}\right]$. This generic expression applies to any hadronic system, including collisions of nuclei with nucleon number $A$ [20], but we will mostly focus below on the proton case for which $A=1$, and $R_{A}=r_{p}$ is its characteristic transverse "radius." From Eq. (3) one obtains

$$
\sigma_{\mathrm{eff}, \mathrm{TPS}}^{2}=\left[\int d^{2} b T_{A A}^{3}(\mathbf{b})\right]^{-1}=\frac{\left(4 \pi R_{A}^{2}\right)^{2}(3 n+1)}{A^{6}(n+1)^{3}} .
$$

Instead of an expression that depends on $R_{A}$, it is more convenient to express the effective TPS cross sections as a 
function of the experimentally determined $\sigma_{\text {eff,DPs }}$ parameter. From [20]

$$
\int d^{2} b T_{A A}^{2}(\mathbf{b})=\frac{A^{4}(n+1)^{2}}{4 \pi R_{A}^{2}(2 n+1)}=A^{2} T_{A A}(0) \frac{n+1}{2 n+1},
$$

one obtains, via Eqs. (3) and (4), the relationship between $\sigma_{\text {eff,TPS }}$ and $\sigma_{\text {eff,DPS: }}$

$$
\sigma_{\mathrm{eff}, \mathrm{TPS}}^{2}=\frac{A^{2}(3 n+1)(n+1)}{(2 n+1)^{2}} \sigma_{\mathrm{eff}, \mathrm{DPS}}^{2},
$$

which for a proton-proton collision reads

$$
\sigma_{\mathrm{eff}, \mathrm{TPS}}^{2}=\frac{(3 n+1)(n+1)}{(2 n+1)^{2}} \sigma_{\mathrm{eff}, \mathrm{DPS}}^{2} .
$$

For the distributions given by Eq. (5) with exponents $n=0-\infty$, one obtains $\sigma_{\text {eff,TPS }}=[1-0.87] \times \sigma_{\text {eff,DPS }}$; i.e., the effective TPS and DPS cross sections are numerically very similar. This result holds also for more realistic hadron profiles. Indeed, modern $p p$ Monte Carlo (MC) event generators, such as PYTHIA [21], often parametrize the $p p$ overlap function in the form

$$
T(\mathbf{b})=\frac{m}{2 \pi r_{p}^{2} \Gamma(2 / m)} \exp \left[-\left(b / r_{p}\right)^{m}\right]
$$

normalized to one, where $\Gamma(2 / m)$ is the gamma function. The exponent $m$ depends on the MC "tune" obtained from fits to the measured $p p$ "underlying event" and various DPS cross sections [14]. It varies between a pure Gaussian $(m=2)$ to more peaked exponential-like $(m=0.7,1)$ distributions. From the corresponding integrals of the square and cube of $T(\mathbf{b})$,

$$
\int d^{2} b T^{2}(\mathbf{b})=\frac{m}{2 \pi r_{p}^{2} \Gamma(2 / m) 2^{2 / m}}
$$

and

$$
\int d^{2} b T^{3}(\mathbf{b})=\left[\frac{m}{2 \pi r_{p}^{2} \Gamma(2 / m)}\right]^{2} \frac{1}{3^{2 / m}},
$$

we obtain, via Eqs. (3) and (4),

$$
\sigma_{\text {eff,TPS }}=(3 / 4)^{1 / m} \sigma_{\text {eff,DPS }},
$$

which is again independent of the exact numerical value of the proton radius $r_{p}$, but depends on the overall shape of its transverse profile characterized by the exponent $m$. For typical PYTHIA $m=0.7,1,2$ exponents tuned from experimental data [14], one obtains $\sigma_{\text {eff,TPS }}=[0.66,0.75$, $0.87] \times \sigma_{\text {eff,DPS }}$, respectively. An alternative phenomenologically motivated description of the proton profile is given by the dipole fit of the two-gluon form factor in the momentum representation [22]

$$
F_{2 g}(\mathbf{q})=1 /\left(q^{2} / m_{g}^{2}+1\right)^{2},
$$

where the gluon mass $m_{g}$ parameter characterizes its transverse momentum $q$ distribution, and the transverse density is obtained from its Fourier transform: $f(\mathbf{b})=$ $\int e^{-i \mathbf{b} \cdot \mathbf{q}} F_{2 g}(\mathbf{q})\left[d^{2} q /(2 \pi)^{2}\right]$. Such a proton profile is used in other common $p p$ MC generators, such as HERWIG [17]. The corresponding DPS and TPS effective cross sections read [11]

$$
\sigma_{\mathrm{eff}, \mathrm{DPS}}=\left[\int F_{2 g}^{4}(q) \frac{d^{2} q}{(2 \pi)^{2}}\right]^{-1}=\frac{28 \pi}{m_{g}^{2}}
$$

and

$$
\begin{aligned}
\sigma_{\mathrm{eff}, \text { TPS }}^{2}= & {\left[\int(2 \pi)^{2} \delta\left(\mathbf{q}_{\mathbf{1}}+\mathbf{q}_{\mathbf{2}}+\mathbf{q}_{\mathbf{3}}\right) F_{2 g}\left(\mathbf{q}_{\mathbf{1}}\right)\right.} \\
& \times F_{2 g}\left(\mathbf{q}_{\mathbf{2}}\right) F_{2 g}\left(\mathbf{q}_{\mathbf{3}}\right) F_{2 g}\left(-\mathbf{q}_{\mathbf{1}}\right) F_{2 g}\left(-\mathbf{q}_{\mathbf{2}}\right) \\
& \left.\times F_{2 g}\left(-\mathbf{q}_{\mathbf{3}}\right) \frac{d^{2} q_{1}}{(2 \pi)^{2}} \frac{d^{2} q_{2}}{(2 \pi)^{2}} \frac{d^{2} q_{3}}{(2 \pi)^{2}}\right]^{-1} .
\end{aligned}
$$

Numerically integrating the latter and combining it with Eq. (8), we obtain $\sigma_{\text {eff,TPS }}=0.83 \times \sigma_{\text {eff,DPS}}$, quite close to the value obtained for the Gaussian $p p$ overlap function.

We note that in order to reproduce the experimentally measured $\sigma_{\text {eff,DPS }} \simeq 15 \mathrm{mb}$, one should fix the characteristic proton "size" to $r_{p} \simeq 0.11,0.24,0.49 \mathrm{fm}$ for $p p$ overlaps of the form given by Eq. (6) with $m=0.7,1$, 2; to $r_{p} \simeq 0.35,0.40,0.46 \mathrm{fm}$ for exponents $n=0,1,2$ as defined in Eq. (5); and to $r_{g}=1 / m_{g} \simeq 0.13 \mathrm{fm}$ in the case of the two-gluon dipole fit Eq. (7). Despite the wide range of effective proton radius parameters derived, one of the main conclusion of this study is the robustness of the $\sigma_{\text {eff,TPS }} \simeq \sigma_{\text {eff,DPS }}$ result. Indeed, the average and standard deviation of all typical parton transverse profiles considered here yields

$$
\sigma_{\text {eff,TPS }}=k \times \sigma_{\text {eff,DPS }}, \quad \text { with } \quad k=0.82 \pm 0.11 ;
$$

i.e., for the typical $\sigma_{\text {eff,DPS }} \simeq 15 \pm 5$ values extracted from a wide range of DPS measurements at Tevatron [13] and LHC [5,13-16], we finally obtain

$$
\sigma_{\text {eff }, \mathrm{TPS}}=12.5 \pm 4.5 \mathrm{mb} \text {. }
$$

The experimental observation of triple parton scatterings in $p p$ collisions requires perturbatively calculable processes with not too high energy (mass) scales so that their SPS cross section are not much smaller than $\mathcal{O}(1 \mu \mathrm{b})$ since, 

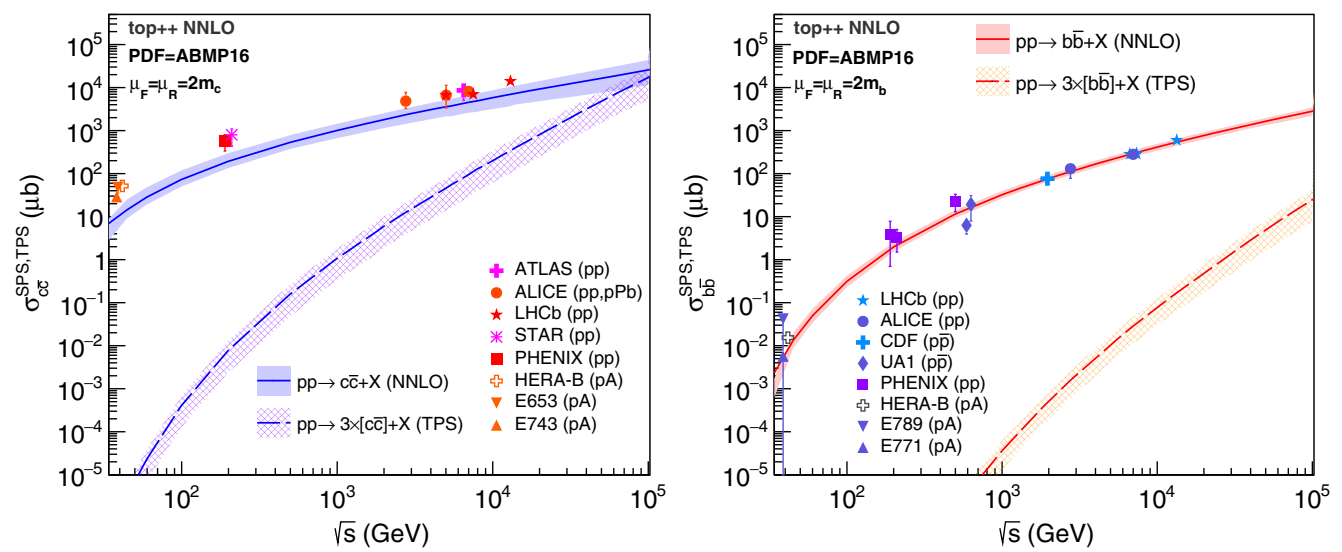

FIG. 1. Total charm (left) and bottom (right) cross sections in $p p$ collisions as a function of c.m. energy, in single parton (solid line) and triple parton (dashed line) scatterings. Bands around curves indicate scale, PDF (and $\sigma_{\text {eff,TPS }}$, in the case of $\sigma^{\mathrm{TPS}}$ ) uncertainties added in quadrature. The symbols are experimental data collected in Ref. [26].

otherwise, their corresponding TPS cross sections (which go as the cube of the SPS values) are extremely reduced. Indeed, according to Eq. (1) with the data-driven estimate Eq. (9), a triple-hard process $p p \rightarrow a a a$, with SPS cross sections $\sigma_{p p \rightarrow a}^{\mathrm{SPS}} \approx 1 \mu \mathrm{b}$, has a very small TPS cross section $\sigma_{p p \rightarrow a a a}^{\mathrm{TPS}} \approx 1 \mathrm{fb}$, already without accounting for extra reducing factors from decay branching ratios and experimental acceptances and reconstruction inefficiencies. Promising processes to probe TPS, with not too small pQCD cross sections, are inclusive charm $p p \rightarrow c \bar{c}+X$ and bottom $p p \rightarrow b \bar{b}+X$ production. These processes are dominated by gluon-gluon fusion, $g g \rightarrow Q \bar{Q}$, and at high energies receive contributions from scatterings at very small $x$, for which one can expect a non-negligible probability of DPS [23-25] and TPS in their total inclusive production.

The TPS heavy-quark cross sections are computed via Eq. (1) for $m=1$, i.e., $\sigma_{p p \rightarrow Q \bar{Q}}^{\mathrm{TPS}}=\left(\sigma_{p p \rightarrow Q \bar{Q}}^{\mathrm{SPS}}\right)^{3} /\left(6 \sigma_{\mathrm{eff}, \mathrm{TPS}}^{2}\right)$, with $\sigma_{\text {eff,TPS }}$ given by Eq. (9), and $\sigma_{p p \rightarrow Q \bar{Q}}^{\mathrm{SPS}}$ calculated via Eq. (2) at NNLO accuracy using a modified version [26] of the TOP++ (v2.0) code [27], with $N_{f}=3,4$ light flavors, heavy-quark pole masses at $m_{c, b}=1.67,4.66 \mathrm{GeV}$, default renormalization and factorization scales $\mu_{R}=\mu_{F}=2 m_{c, b}$, and using the ABMP16 PDFs [28]. The NNLO calculations increase the total SPS heavy-quark cross sections by up to $20 \%$ at LHC energies compared to NLO results [29,30],

TABLE I. Total charm and bottom SPS (NNLO) and TPS cross sections (in mb) in $p p$ at LHC and FCC with scales (sc), PDF, and total (quadratic, including $\sigma_{\text {eff,TPS }}$ ) uncertainties.

\begin{tabular}{lcc}
\hline \hline Final state & $\sqrt{s}=14 \mathrm{TeV}$ & $\sqrt{s}=100 \mathrm{TeV}$ \\
\hline$\sigma(c \bar{c}+X)$ & $7.1 \pm 3.5_{\mathrm{sc}} \pm 0.3_{\mathrm{PDF}}$ & $25.0 \pm 16.0_{\mathrm{sc}} \pm 1.3_{\mathrm{PDF}}$ \\
$\sigma(c \bar{c} c \bar{c} c \bar{c}+X)$ & $0.39 \pm 0.28_{\mathrm{tot}}$ & $16.7 \pm 11.8_{\mathrm{tot}}$ \\
$\sigma(b \bar{b}+X)$ & $0.56 \pm 0.09_{\mathrm{sc}} \pm 0.01_{\mathrm{PDF}}$ & $2.8 \pm 0.6_{\mathrm{sc}} \pm 0.1_{\mathrm{PDF}}$ \\
$\sigma(b \bar{b} b \bar{b} b \bar{b}+X)$ & $\left(0.19 \pm 0.12_{\mathrm{tot}}\right) 10^{-3}$ & $\left(24 \pm 17_{\mathrm{tot}}\right) 10^{-3}$ \\
\hline \hline
\end{tabular}

reaching a better agreement with the experimental data, and featuring much reduced scale uncertainties $( \pm 50 \%, \pm 15 \%$ for $c \bar{c}, b \bar{b}$ ) [26]. Figure 1 shows the resulting total SPS and TPS cross sections for charm and bottom production over $\sqrt{s}=35 \mathrm{GeV}-100 \mathrm{TeV}$, and Table I collects a few values with associated uncertainties for nominal LHC and FCC energies. The PDF uncertainties are obtained from the corresponding 28 eigenvalues of the ABMP16 set. The dominant uncertainty comes from the theoretical scale dependence, which is estimated by modifying $\mu_{R}$ and $\mu_{F}$ within a factor of 2 . The TPS cross sections are small but rise fast with $\sqrt{s}$, as the cube of the SPS cross sections. Although triple- $b \bar{b}$ cross sections remain quite small and reach only about $1 \%$ of the inclusive bottom cross section at the FCC $(\sqrt{s}=100 \mathrm{TeV})$, triple- $c \bar{c}$ production from three independent parton scatterings amounts to $5 \%$ of the inclusive charm yields at the $\operatorname{LHC}(\sqrt{s}=14 \mathrm{TeV})$ and to more than half of the total charm cross section at the FCC. Since the total $p p$ inelastic cross section at $\sqrt{s}=100 \mathrm{TeV}$ is $\sigma_{p p} \simeq 105 \mathrm{mb}$ [31], charm-anticharm triplets are expected to be produced in about $15 \%$ of the $p p$ collisions at these energies.

The study presented here demonstrates that triple parton scatterings are a non-negligible source of perturbative particle production in $p p$ collisions at increasingly higher energies. The formulas derived here allow one to easily estimate the TPS yields for any final state of interest. Using accurate NNLO predictions for heavy-quark production in single parton scatterings, we have shown that the production cross section of three $c \bar{c}$ pairs from three separate parton interactions is in principle observable at the LHC, and approaches the total charm cross section at $\sqrt{s} \approx 100 \mathrm{TeV}$.

Discussions with A. P. Kryukov and M. A. Malyshev on TPS, and with M. Cacciari, M. Czakon, A. Mitov, S. Moch, and G. Salam on NNLO heavy-quark calculations are gratefully acknowledged. 
[1] M. Diehl, D. Ostermeier, and A. Schafer, J. High Energy Phys. 03 (2012) 089.

[2] P. Bartalini et al., arXiv:1111.0469.

[3] H. Abramowicz et al., arXiv:1306.5413.

[4] S. Bansal et al., arXiv:1410.6664.

[5] R Astalos et al., arXiv:1506.05829.

[6] DESY Report No. DESY-PROC-2016-01, edited by H. Jung, D. Treleani, M. Strikman, and N. van Buuren.

[7] D. d'Enterria, G. Kh. Eyyubova, V. L. Korotkikh, I. P. Lokhtin, S. V. Petrushanko, L. I. Sarycheva, and A. M. Snigirev, Eur. Phys. J. C 66, 173 (2010).

[8] G. Calucci and D. Treleani, Phys. Rev. D 83, 016012 (2011).

[9] G. Calucci and D. Treleani, Phys. Rev. D 79, 074013 (2009); 79, 074013 (2009); 80, 054025 (2009); 86, 036003 (2012).

[10] E. Maina, J. High Energy Phys. 09 (2009) 081.

[11] A. M. Snigirev, Phys. Rev. D 94, 034026 (2016).

[12] M. Mangano et al., arXiv:1607.01831.

[13] (CDF Collaboration), Phys. Rev. D 56, 3811 (1997).

[14] (CMS Collaboration), Eur. Phys. J. C 76, 155 (2016).

[15] (ATLAS Collaboration), J. High Energy Phys. 11 (2016) 110.

[16] (LHCb Collaboration), J. High Energy Phys. 07 (2016) 052.

[17] M. H. Seymour and A. Siodmok, J. High Energy Phys. 10 (2013) 113.

[18] H. S. Shao, Comput. Phys. Commun. 184, 2562 (2013).
[19] J. P. Lansberg and H. S. Shao, Phys. Lett. B 751, 479 (2015).

[20] D. d'Enterria and A. M. Snigirev, Phys. Lett. B 718, 1395 (2013); 727, 157 (2013); arXiv:1612.08112.

[21] T. Sjöstrand, S. Mrenna, and P. Z. Skands, Comput. Phys. Commun. 178, 852 (2008).

[22] B. Blok, Y. Dokshitzer, L. Frankfurt, and M. Strikman, Phys. Rev. D 83, 071501 (2011).

[23] M. Luszczak, R. Maciula, and A. Szczurek, Phys. Rev. D 85, 094034 (2012).

[24] A. V. Berezhnoy, A. K. Likhoded, A. V. Luchinsky, and A. A. Novoselov, Phys. Rev. D 86, 034017 (2012).

[25] E. R. Cazaroto, V. P. Goncalves, and F. S. Navarra, Phys. Rev. D 88, 034005 (2013).

[26] David d'Enterria (to be published).

[27] M. Czakon, P. Fiedler, and A. Mitov, Phys. Rev. Lett. 110, 252004 (2013).

[28] S. Alekhin et al., arXiv:1609.03327.

[29] M. Cacciari, S. Frixione, N. Houdeau, M. L. Mangano, P. Nason, and G. Ridolfi, J. High Energy Phys. 10 (2012) 137.

[30] M. L. Mangano, P. Nason, and G. Ridolfi, Nucl. Phys. B373, 295 (1992).

[31] D. d'Enterria and T. Pierog, J. High Energy Phys. 08 (2016) 170. 